\title{
Paclitaxel and carboplatin chemotherapy after platinum-based chemotherapy and pembrolizumab for metastatic urothelial carcinoma
}

\author{
NOBUKI FURUBAYASHI ${ }^{1}$, YOSHIFUMI HORI ${ }^{2}$, FUTOSHI MOROKUMA $^{3}$, \\ TOSHIHISA TOMODA ${ }^{4}$, TAKAHITO NEGISHI ${ }^{1}$, TOMOHIRO INOUE $^{1}$, MASATOSHI KUMAGAI $^{1}$, \\ KENTARO KUROIWA $^{2}$, NORIAKI TOKUDA ${ }^{3}$ and MOTONOBU NAKAMURA ${ }^{1}$
}

\author{
${ }^{1}$ Department of Urology, National Hospital Organization Kyushu Cancer Center, Fukuoka 811-1395; \\ ${ }^{2}$ Department of Urology, Miyazaki Prefectural Miyazaki Hospital, Miyazaki 880-8510; ${ }^{3}$ Department of Urology, \\ Saga-ken Medical Centre Koseikan, Saga 840-8571; ${ }^{4}$ Department of Urology, Oita Prefectural Hospital, Oita 870-8511, Japan
}

Received October 15, 2020; Accepted February 15, 2021

DOI: $10.3892 / \mathrm{mco} .2021 .2253$

\begin{abstract}
Pembrolizumab has been available for the treatment of radical resectable urothelial carcinoma (UC) when it is exacerbated after chemotherapy since December 2017 in Japan. However, the efficacy of chemotherapy for cases progressing after pembrolizumab is unclear. The present study compared the outcomes and toxicities in patients with metastatic UC after failure of platinum-based chemotherapy and pembrolizumab, who were selected to receive paclitaxel and carboplatin (TC) chemotherapy, with those in patients who received the best supportive care (BSC). A total of 36 patients received pembrolizumab for metastatic UC at four institutions between January 2018 and August 2019. Of the 21 patients who progressed after pembrolizumab, 7 received TC chemotherapy (TC group) and 14 selected BSC (BSC group). The median observation period was 4.1 months. The 7 aforementioned patients who received TC chemotherapy (4 male and 3 female; median age, 62 years; range, 57-79 years) were analyzed in the present study. The ECOG performance status was 0 in three patients, 1 in one patient, 2 in two patients and 3 in one patient. Two patients had upper urinary tract UC, two had bladder UC and three had both types of UC. Six patients had visceral metastasis. The number of chemotherapy regimens before pembrolizumab was one in four patients, two in two patients and three in one patient. The objective response rate was $28.6 \%$ (partial response, 2 patients; stable disease, 4 patients; progressive disease, 1 patient), the median progression-free survival time was 3.4 months and the median overall survival time was 10.9 months (vs. 2.7 months
\end{abstract}

Correspondence to: Dr Nobuki Furubayashi, Department of Urology, National Hospital Organization Kyushu Cancer Center, Notame 3-1-1, Minami, Fukuoka 811-1395, Japan

E-mail: furubayashi-jua@umin.ac.jp

Key words: urothelial carcinoma, platinum-based chemotherapy, pembrolizumab, paclitaxel, carboplatin in $\mathrm{BSC}$ group; $\mathrm{P}=0.0156)$. Although grade $\geq 3$ adverse events developed in five patients, there were no treatment-associated deaths. The present results suggested that TC chemotherapy may be a preferred option for patients who require aggressive treatment after the failure of platinum-based chemotherapy and pembrolizumab.

\section{Introduction}

Locally advanced or metastatic urothelial carcinoma (UC) is an incurable disease with a poor long-term survival (1). Until recently, cytotoxic chemotherapy was the only treatment available for advanced UC, and platinum-based chemotherapy had been the gold-standard treatment $(2,3)$. However, platinum resistance develops rapidly, and nearly $80 \%$ of cases will relapse. The prognosis is extremely poor after failure of platinum-based chemotherapy. Second-line chemotherapy regimens with various single agents and combinations of agents have been reported, but the prognosis is extremely poor $(1,4,5)$.

Pembrolizumab, a humanized monoclonal antibody targeting programmed death receptor-1 (PD-1), was a second-line treatment for platinum-refractory patients with significantly longer overall survival (approximately 3 months) and a lower incidence of treatment-related adverse events in comparison to chemotherapy in the KEYNOTE-045 phase III trial (6). This open-label, international, phase 3 trial also showed that the objective response rate was significantly higher in the pembrolizumab group $(21.1 \%)$ than in the chemotherapy group $(11.4 \%)(\mathrm{P}=0.001)$. Since December 2017, pembrolizumab has been approved in Japan as a second-line treatment for radically unresectable UC, that has worsened after chemotherapy (7). However, the objective response rate in the pembrolizumab group was not still satisfactory at $21.1 \%$, so most patients develop progressive disease after pembrolizumab and choose best support care (BSC) or chemotherapy if they desire aggressive treatment, as there is no standard care after platinum-based chemotherapy and pembrolizumab. 
However, to the best of our knowledge, there are no published studies describing the results of paclitaxel and carboplatin (TC) chemotherapy alone in metastatic UC when administered to patients with progression during treatment with platinum-based chemotherapy and antibodies targeting PD-1 (pembrolizumab). We retrospectively compared the outcomes and toxicities of patients with metastatic UC after failure of platinum-based chemotherapy and pembrolizumab who selected TC chemotherapy with those in patients who received BSC.

\section{Patients and methods}

Patients and methods. Thirty-six patients received pembrolizumab for metastatic UC at four institutions from January 2018 to August 2019. Of the 21 patients who progressed after pembrolizumab, 7 received TC chemotherapy (TC group), and 14 selected the BSC (BSC group). All patients with UC had histopathologically diagnosed and radiologically confirmed disease progression after platinum-based chemotherapy and pembrolizumab (8). The TC regimen, paclitaxel $\left(175 \mathrm{mg} / \mathrm{m}^{2}\right)$ and carboplatin (area under the curve: 5), was administered intravenously on day 1 every 21 days and was repeated until disease progression or unacceptable adverse events occurred. Tumors were generally measured by computed tomography before and after every 2-3 cycles of TC chemotherapy. Decisions on adverse events were made in accordance with the Common Terminology Criteria for Adverse Events, version 5.0 (9). The tumor response was assessed as the best response according to the Response Evaluation Criteria in Solid Tumors, version 1.1 (10).

All of the patients provided their written informed consent to participate in this study, and the study protocol was approved by the Ethics Committee of the Kyushu Cancer Center (Fukuoka, Japan).

Statistical analyses. The statistical analyses were carried out using the $\mathrm{JMP}^{\circledR}$ Pro, version 14.2.0 software package (SAS Institute, Inc., Cary, NC, USA). The objective response rate is defined as the proportion of patients who achieve a partial or complete response to TC chemotherapy. The progression-free survival (PFS) in the TC group was calculated from the day on which chemotherapy was started until the date when patients who were alive and without disease progression or who were lost to follow-up had their data censored at the time of the final tumor assessment. In the BSC group, in general no computed tomography findings were examined after patients selected BSC. Therefore, the PFS was not calculated in the BSC group. The overall survival (OS) was calculated from the day that BSC was selected or the day on which chemotherapy was started until the date of the last follow-up examination or death from any cause. The Mann-Whitney U test was used to assess the differences between the BSC and TC groups. The Kaplan-Meier method was used to evaluate progression-free survival (PFS) and OS, and the differences between the BSC and TC groups was determined by the log-rank test. P-values of $<0.05$ were considered to indicate statistical significance.

\section{Results}

Patient characteristics. The clinical characteristics are listed in Table I. Twenty-one patients were enrolled in this study (male, $\mathrm{n}=17,91 \%$; median age, 70 years; range, $57-85$ years). The patients selected BSC or TC chemotherapy after the failure of platinum-based chemotherapy and pembrolizumab for UC. Eight patients had bladder UC, seven had upper urinary tract UC, and six had both types of UC. All patients except one had visceral metastasis. With regard to the treatment after pembrolizumab, 14 patients $(66.7 \%)$ selected BSC, and $7(33.3 \%)$ received TC chemotherapy. The median time from the fist-line chemotherapy treatment was 13.9 months (95\% CI, 12.0-27.2 months). There were no statistically significant differences in any characteristics between the BSC and TC chemotherapy groups. Regarding the regimens administered prior to TC or BSC, 13 patients received gemcitabine + cisplatin $(\mathrm{GC})$ and pembrolizumab, 2 patients received GC, methotrexate + vinblastine + doxorubicin + cisplatin (MVAC) and pembrolizumab, 2 patients received GC, TC, MVAC and pembrolizumab, 2 patients received GC, avelumab and pembrolizumab, 1 patient received gemcitabine+carboplatin (GCBDCA), GC, gemcitabine + paclitaxel and pembrolizumab, and 1 patient received GC, GCBDCA, G and pembrolizumab.

The PFS of TC chemotherapy and OS according to treatments after pembrolizumab. The PFS of TC chemotherapy after pembrolizumab was 3.4 months [95\% confidence interval (CI), 0.6-6.6 months] (Fig. 1). The OS according to treatment after pembrolizumab is shown in Fig. 2. The median OS for BSC was 2.7 months (95\% CI, 0.6-4.8 months), and the median OS for TC was 10.9 months (95\% CI, 0.9-12.7 months). The estimated OS rate was $75 \%$ at 6 months and $30 \%$ at 12 months in the TC chemotherapy group.

A log-rank test revealed a statistically significant difference in OS between BSC and TC $(\mathrm{P}=0.0156)$.

The response analysis and toxicities in patients who received TC chemotherapy after pembrolizumab. The objective tumor responses are shown in Table II. Among the 7 patients who received TC chemotherapy after pembrolizumab, a complete response (CR) was not confirmed in any patients, while 2 patients $(28.6 \%)$ showed a partial response (PR), with an objective response rate of $28.6 \%$. The disease control rate (defined by the achievement of CR, PR or SD) was $85.7 \%$, which was defined as a tumor response of CR, PR or stable disease [SD].

Table III shows the adverse events associated with TC chemotherapy after pembrolizumab. Myelosuppression was the most common toxicity. Grade $\geq 3$ thrombocytopenia and neutropenia occurred in 3 patients $(42.9 \%)$, while febrile neutropenia was observed in 2 patients $(28.6 \%)$; no patients showed severe infection. Grade 3 anemia occurred in 1 patient $(14.3 \%)$. With regard to non-hematological toxicities, grade 3 fatigue developed in 1 patient. All other toxicities were less than grade 3 in severity, and no immune-related adverse events occurred. No treatment-related deaths occurred among the seven patients.

\section{Discussion}

This study represents the first specific report of outcomes focusing on TC chemotherapy after the failure of platinum-based chemotherapy and pembrolizumab for advanced 
Table I. Clinicopathological characteristics of patients with urothelial carcinoma $(n=21)$.

\begin{tabular}{|c|c|c|c|}
\hline Characteristics & $\begin{array}{l}\text { Paclitaxel and carboplatin } \\
\text { chemotherapy }(n=7)\end{array}$ & $\begin{array}{l}\text { Best supportive care } \\
\qquad(\mathrm{n}=14)\end{array}$ & P-value \\
\hline Sex, male, n (\%) & $4(57)$ & $13(93)$ & 0.054 \\
\hline Median age (range), years & $62(57-79)$ & $70(58-85)$ & 0.108 \\
\hline ECOG PS, n (\%) & & & 0.546 \\
\hline 0 & $3(43)$ & $3(21)$ & \\
\hline 1 & $1(14)$ & $4(29)$ & \\
\hline$\geq 2$ & $3(43)$ & $7(50)$ & \\
\hline Primary tumor site, $\mathrm{n}(\%)$ & & & 0.874 \\
\hline Bladder & $2(28)$ & $6(43)$ & \\
\hline Upper urinary tract & $2(28)$ & $5(36)$ & \\
\hline Bladder + upper urinary tract & $3(43)$ & $3(21)$ & \\
\hline No. of chemotherapy before pembrolizumab & & & 0.931 \\
\hline 1 & $4(57)$ & $9(64)$ & \\
\hline 2 & $2(28)$ & $2(15)$ & \\
\hline 3 & $1(14)$ & $3(21)$ & \\
\hline $\begin{array}{l}\text { Median time from first-line chemotherapy, } \\
\text { months }(95 \% \mathrm{CI})\end{array}$ & $11.8(4.7-31.1)$ & $15.8(10.4-31.7)$ & 0.371 \\
\hline Metastasis sites, $\mathrm{n}(\%)$ & & & 0.129 \\
\hline Lymph nodes only & $1(14)$ & $0(0)$ & \\
\hline Visceral disease & $6(86)$ & $14(100)$ & \\
\hline
\end{tabular}

ECOG PS, Eastern Cooperative Oncology Group Performance Status.

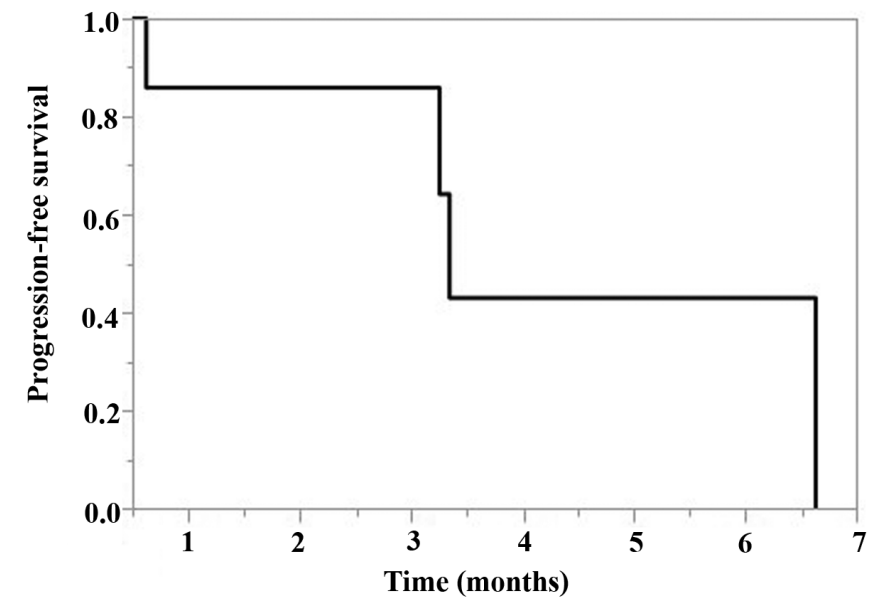

Figure 1. Progression-free survival of paclitaxel and carboplatin chemotherapy group $(n=7)$.

UC patients. In patients with metastatic UC who had previously been treated with both platinum-based chemotherapy and pembrolizumab, TC chemotherapy led to a $28.6 \%$ objective response rate, and the OS was 10.9 months. This systematic review of the efficacy of chemotherapy in the setting after platinum-based chemotherapy and immune checkpoint inhibitor treatment suggests that anti-PD1 may have a delayed synergistic effect on subsequent cytotoxic therapy.

There is currently no data on chemotherapy for advanced UC after pembrolizumab. There is also no data on what is being done in the post-treatment of pembrolizumab arm in

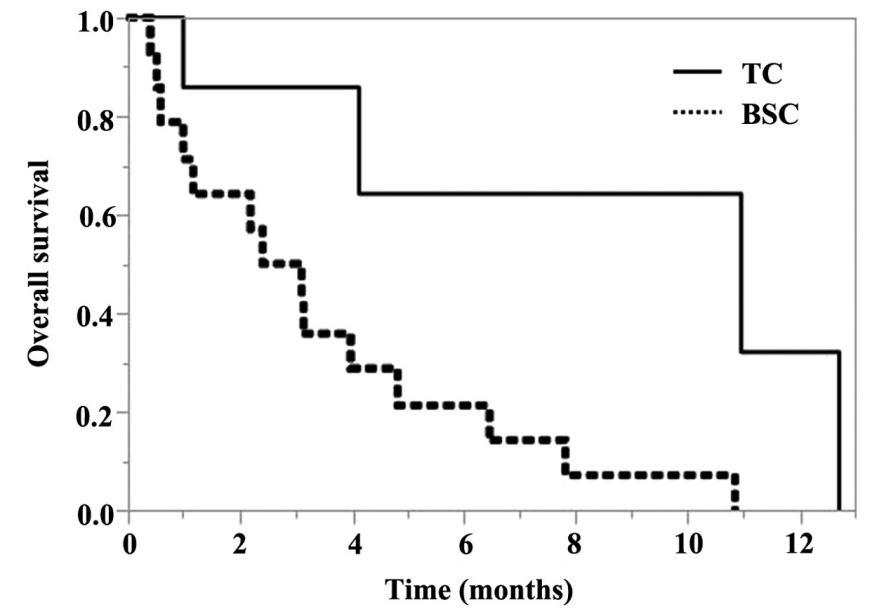

Figure 2. Overall survival according to the treatment. TC, paclitaxel and carboplatin; BSC, best supportive care.

KEYNOTE-045 study (11). Therefore, the chemotherapy regimen that has been used in the second-line setting before pembrolizumab is expected to be able to be administered to patients who desire aggressive treatment after pembrolizumab failure. Our previous study reviewed the tolerability and efficacy of TC therapy as second-line therapy for UC that is resistant to gemcitabine and cisplatin (GC) as a primary chemotherapy regimen (12). We reported that the median OS was 12.7 months (95\% CI, 3.1-25.4 months), the objective response rate (CR $6.2 \%$, PR $12.5 \%$ ) was $18.7 \%$, and the disease control rate was $56.2 \%$ in patients who received TC 
Table II. Analysis of the responses of patients who received paclitaxel and carboplatin chemotherapy $(n=7)$.

\begin{tabular}{lcc}
\hline Response & No. of patients & Response rate, \% \\
\hline CR & 0 & 0 \\
PR & 2 & 28.6 \\
SD & 4 & 57.1 \\
PD & 1 & 14.3 \\
Overall response rate (CR + PR) & 2 & 28.6 \\
Disease control rate (CR + PR + SD) & 6 & 85.7 \\
\hline
\end{tabular}

$\mathrm{CR}$, complete response; $\mathrm{PR}$, partial response; $\mathrm{SD}$, stable disease; $\mathrm{PD}$, progressive disease.

Table III. Toxicities in patients treated with paclitaxel and carboplatin chemotherapy $(n=7)$.

\begin{tabular}{|c|c|c|c|c|c|}
\hline Adverse events & Grade $1, \mathrm{n}$ & Grade 2, n & Grade $3, \mathrm{n}$ & Grade 4, n & Grade $\geq 3, \%$ \\
\hline Thrombocytopenia & 0 & 1 & 3 & 0 & 42.9 \\
\hline Neutropenia & 0 & 0 & 1 & 2 & 42.9 \\
\hline Febrile neutropenia & NA & NA & 2 & 0 & 28.6 \\
\hline Anemia & 1 & 1 & 1 & 0 & 14.3 \\
\hline Fatigue & 0 & 1 & 1 & 0 & 14.3 \\
\hline Neuropathy & 1 & 1 & 0 & 0 & 0.0 \\
\hline Rash maculo-papular & 0 & 1 & 0 & NA & 0.0 \\
\hline Alopecia & 0 & 3 & NA & NA & NA \\
\hline
\end{tabular}

NA, not applicable.

chemotherapy as a second-line regimen. In the present study, we reviewed the tolerability and efficacy of TC chemotherapy after the failure of platinum-based chemotherapy and pembrolizumab for advanced UC patients. It revealed that the median OS was 10.9 months (95\% CI, 0.9-12.7 months), the objective response rate was $28.6 \%$, and the disease control rate was $85.7 \%$ in patients who received TC chemotherapy after the failure of platinum-based chemotherapy and pembrolizumab. These data may suggest a lack of cross-resistance between chemotherapy and immune checkpoint inhibitors in metastatic UC. In addition, chemotherapy may be more effective after immune checkpoint inhibitors, even as a third-line treatment. The safety was virtually the same as in our previous report, and no new safety signals were recognized. This result also shows that TC chemotherapy can be safely administered even after immune checkpoint inhibitors.

Exposure to vaccine-based immunotherapy followed by chemotherapy has been previously reported to be associated with an improved response to treatment in other types of cancer (13-18). Similarly, other reports of patients with advanced tumors have shown improved response rates and improved survival in patients who received chemotherapy administered after various vaccine-based treatment strategies $(17,18)$. Although chemotherapy has historically been considered immunosuppressive, several mechanisms have been proposed for the enhancement of tumor immunity with certain agents, such as increasing neoantigen presentation and cell recognition, abrogating myeloid-derived suppressor cell and T-regulatory cell activity and enhancing cross-priming and promoting anti-tumor $\mathrm{CD}^{+}{ }^{+} \mathrm{T}$-cell phenotype (19). A recent phase 2 randomized trial report on non-small-cell lung cancer supports there is a positive interaction between chemotherapy and checkpoint inhibitors. This trial included 123 patients who were randomized to receive front-line carboplatin and pemetrexed with or without pembrolizumab; the combination group showed a favorable objective response rate (55\% vs. $29 \%$ ) and PFS (13.0 vs. 8.9 months, $\mathrm{P}=0.01)$ (20). In addition, it was also reported that the confirmed objective response rate to single-agent chemotherapy after immunotherapy exposure was higher than that described in historical data from the pre-anti-PD1 era and was similar to the objective response rate to first-line platinum-based chemotherapy in non-small-cell lung cancer patients (21). A previous study compared the response rates of patients with metastatic urothelial carcinoma who received third-line chemotherapy treatment (after chemotherapy and immune checkpoint inhibitors) and second-line chemotherapy treatment (after only immune checkpoint inhibitors) (22). Patients who receive chemotherapy for the first time after immune checkpoint inhibitors showed a high response rate to the chemotherapy (64\%), which suggests that there is no cross-resistance between the two classes of agents. The same appears to apply to patients who have previously received both chemotherapy and immune checkpoint inhibitors. The chemotherapy response rate of $21 \%$ is in line with the expected results in patients who have previously failed chemotherapy without a history of immune checkpoint 
inhibitor exposure (23). Taken together these results suggest that the chemotherapy responses are maintained, regardless of the history of exposure to immune checkpoint inhibitors in cases of metastatic urothelial carcinoma.

In the present study, the efficacy of TC chemotherapy after the failure of platinum-based chemotherapy and pembrolizumab for advanced UC was better than the previously reported efficacy of TC chemotherapy as a second-line regimen for advanced UC showing resistance to GC as a first-line chemotherapy regimen (objective response rate $28.6 \%$ vs. $18.7 \%$ ) (12). These results suggest that synergy exists between immune checkpoint inhibitors and chemotherapy. In a previous study, the duration of pembrolizumab, a PD-1-blocking antibody, in T cells of non-small-cell lung cancer patients was systematically assessed, and complete binding to $\mathrm{T}$ cells was reportedly lost after approximately 20 to 25 weeks (24). Anti-PD1 also may provide a delayed synergistic effect on subsequent cytotoxic therapy and may contribute to improved therapeutic efficacy through the overlap of circulating anti-PD1 and cytotoxic agents. No standard subsequent-line therapy after platinum-based chemotherapy and pembrolizumab has been established in Japan. Erdafitinib (a tyrosine kinase inhibitor of fibroblast growth factor receptor 1-4) and enfortumab vedotin (Nectin-4-directed antibody-drug conjugate) are recommended as the preferred subsequent-line systemic therapy options according to the National Comprehensive Cancer Network guidelines (25-27), but these drugs are not yet approved in Japan. Therefore, we administer TC chemotherapy as salvage chemotherapy for such patients. Paclitaxel is an antimitotic spindle drug that promotes microtubular aggregation and interferes with certain cell functions, including cell mitosis, transport and motility. Single-agent paclitaxel was shown to have an overall response rate of $42 \%$ in previously untreated patients with UC (28) and $70 \%$ when administered in combination with cisplatin (29). Platinum-based agents have also been frequently included in salvage chemotherapy, which is provided even after the failure of a platinum-based regimen; the efficacy of this agent against platinum-resistant disease has been reported $(30,31)$. However, patients with UC often have an impaired renal function due to an advanced age, history of platinum-containing chemotherapy, history of nephrectomy and/or disease-related hydronephrosis. Carboplatin is a less nephrotoxic and emetogenic platinum compound than cisplatin (32); thus, carboplatin is considered a favorable agent for second-line regimens. Therefore, salvage chemotherapy (TC chemotherapy was selected in the present study) after progression on platinum-based chemotherapy and immune checkpoint inhibitors may also be options for treatment in patients with metastatic UC. We previously reported the utility of TC chemotherapy as second-line treatment after the failure of $\mathrm{GC}$ chemotherapy (12), and the efficacy of TC chemotherapy was compared with that of BSC. The present study reported the efficacy of TC chemotherapy after the failure of GC chemotherapy and pembrolizumab and compared its efficacy with that of BSC. Although the present study was a retrospective study, we believe that comparing the results of TC chemotherapy under similar conditions will show that TC therapy can be an effective treatment method even after the failure of GC chemotherapy and pembrolizumab.
Several limitations associated with the present study warrant mention. First, we evaluated the clinical practice data related to the efficacy and tolerability of TC chemotherapy after the failure of platinum-based chemotherapy and pembrolizumab for metastatic UC in a retrospective, non-randomized, trial. Second, the median observation period for the present study was short at 4.1 months. When comparing OS and BSC, a longer observation period might be necessary. However, the overall survival (natural history) after the failure of platinum-based chemotherapy and pembrolizumab in patients who selected BSC has rarely been reported. The present study showed that the progression-free survival was not very long, even if patients selected TC chemotherapy. Therefore, extending the observation period is expected to be difficult. Third, the population of the current study was relatively small population; thus, further studies are needed to confirm our data in a larger study population. In the present analysis, in a small study population, TC chemotherapy achieved a $28.6 \%$ objective response rate and the toxicity profile was tolerable as third-line or beyond treatment in patients with advanced or metastatic UC who had previously received platinum-based chemotherapy and pembrolizumab. TC chemotherapy is a suitable option for patients who desire aggressive treatment after failure of platinum-based chemotherapy and pembrolizumab in advanced or metastatic UC.

\section{Acknowledgements}

Not applicable.

\section{Funding}

No funding was received.

\section{Availability of data and materials}

The datasets used and/or analyzed during the current study are available from the corresponding author on reasonable request.

\section{Authors' contributions}

NF, YH and MN conceived and designed the analysis. All authors acquired the data. FM, TN, TI and MK confirmed the authenticity of the data and analyzed the data. NF and $\mathrm{YH}$ drafted the manuscript. TT, KK, NT and MN acquired the data, assisted with statistical analysis, supervised the study and critically revised the manuscript for important intellectual content. All authors read and approved the final manuscript.

\section{Ethics approval and consent to participate}

The present study was approved by the Institutional Review Board of the National Hospital Organization Kyushu Cancer Center (approval no. 2014-99). Written informed consent was provided by all patients prior to the study start.

\section{Patient consent for publication}

Not applicable. 


\section{Competing interests}

The authors declare that they have no competing interests.

\section{References}

1. Bellmunt J, Théodore C, Demkov T, Komyakov B, Sengelov L, Daugaard G,Caty A,Carles J,Jagiello-Gruszfeld A, Karyakin O, et al: Phase III trial of vinflunine plus best supportive care compared with best supportive care alone after a platinum-containing regimen in patients with advanced transitional cell carcinoma of the urothelial tract. J Clin Oncol 27: 4454-4461, 2009.

2. Loehrer PJ Sr, Einhorn LH, Elson PJ, Crawford ED, Kuebler P, Tannock I, Raghavan D, Stuart-Harris R, Sarosdy MF, Lowe BA, et al: A randomized comparison of cisplatin alone or in combination with methotrexate, vinblastine, and doxorubicin in patients with metastatic urothelial carcinoma: A cooperative group study. J Clin Oncol 10: 1066-1073, 1992.

3. von der Maase H, Hansen SW, Roberts JT, Dogliotti L, Oliver T, Moore MJ, Bodrogi I, Albers P, Knuth A, Lippert CM, et al: Gemcitabine and cisplatin versus methotrexate, vinblastine, doxorubicin, and cisplatin in advanced or metastatic bladder cancer: Results of a large, randomized, multinational, multicenter, phase III study. J Clin Oncol 18: 3068-3077, 2000.

4. Pronzato P, Vigani A, Pensa F, Vanoli M, Tani F and Vaira F: Second line chemotherapy with ifosfamide as outpatient treatment for advanced bladder cancer. Am J Clin Oncol 20: 519-521, 1997.

5. Vaughn DJ, Broome CM, Hussain M, Gutheil JC and Markowitz AB: Phase II trial of weekly paclitaxel in patients with previously treated advanced urothelial cancer. J Clin Oncol 20: 937-940, 2002

6. Bellmunt J, de Wit R, Vaughn DJ, Fradet Y, Lee JL, Fong L, Vogelzang NJ, Climent MA, Petrylak DP, Choueiri TK, et al: Pembrolizumab as second-line therapy for advanced urothelial carcinoma. N Engl J Med 376: 1015-1026, 2017.

7. Yuasa T, Urakami S and Yonese J: Recent advances in medical therapy for metastatic urothelial cancer. Int J Clin Oncol 23: 599-607, 2018

8. Moch H, Cubilla AL, Humphrey PA, Reuter VE and Ulbright TM: The 2016 WHO classification of tumours of the urinary system and male genital organs-part A: Renal, penile, and testicular tumours. Eur Urol 70: 93-105, 2016.

9. National Cancer Institute: Common Terminology Criteria for Adverse Events (CTCAE). https://ctep.cancer.gov/protocolDevelopment/electronic_applications/ctc.htm\#ctc_50. Last Updated September 21, 2020

10. Eisenhauer EA, Therasse P, Bogaerts J, Schwartz LH, Sargent D, Ford R, Dancey J, Arbuck S, Gwyther S, Mooney M, et al: New response evaluation criteria in solid tumours: Revised RECIST guideline (version 1.1). Eur J Cancer 45: 228-247, 2009.

11. Fradet Y, Bellmunt J, Vaughn DJ, Lee JL, Fong L, Vogelzang NJ, Climent MA, Petrylak DP, Choueiri TK, Necchi A, et al: Randomized phase III KEYNOTE-045 trial of pembrolizumab versus paclitaxel, docetaxel, or vinflunine in recurrent advanced urothelial cancer: Results of $>2$ years of follow-up. Ann Oncol 30: 970-976, 2019

12. Furubayashi N, Negishi T, Yamashita T, Kusano S, Taguchi K, Shimokawa M, Nakamura M: The combination of paclitaxel and carboplatin as second-line chemotherapy can be a preferred regimen for patients with urothelial carcinoma after the failure of gemcitabine and cisplatin chemotherapy. Mol Clin Oncol 7 : 1112-1118, 2017

13. Antonia SJ, Mirza N, Fricke I, Chiappori A, Thompson P, Williams N, Bepler G, Simon G, Janssen W, Lee JH, et al: Combination of p53 cancer vaccine with chemotherapy in patients with extensive stage small cell lung cancer. Clin. Cancer Res 12: 878-887, 2006.

14. Radfar S, Wang Y and Khong HT: Activated CD4 ${ }^{+} \mathrm{T}$ cells dramatically enhance chemotherapeutic tumor responses in vitro and in vivo. J Immunol 183: 6800-6807, 2009.

15. Chiappori AA, Soliman H, Janssen WE, Antonia SJ and Gabrilovich DI: INGN-225: A dendritic cell-based p53 vaccine (Ad.p53-DC) in small cell lung cancer: Observed association between immune response and enhanced chemotherapy effect. Expert Opin Biol Ther 10: 983-991, 2010.
16. Ramakrishnan R, Antonia S and Gabrilovich DI: Combined modality immunotherapy and chemotherapy: A new perspective. Cancer Immunol Immunother 57: 1523-1529, 2008.

17. Gribben JG, Ryan DP, Boyajian R, Urban RG, Hedley ML, Beach K, Nealon P, Matulonis U, Campos S, Gilligan TD, et al: Unexpected association between induction of immunity to the universal tumor antigen CYP1B1 and response to next therapy. Clin Cancer Res 11: 4430-4436, 2005.

18. Wheeler CJ, Das A, Liu G, Yu JS and Black KL: Clinical responsiveness of glioblastoma multiforme to chemotherapy after vaccination. Clin Cancer Res 10: 5316-5326, 2004.

19. Emens LA and Middleton G: The interplay of immunotherapy and chemotherapy: Harnessing potential synergies. Cancer Immunol Res 3: 436-443, 2015.

20. Langer CJ, Gadgeel SM, Borghaei H, Papadimitrakopoulou VA, Patnaik A, Powell SF, Gentzler RD, Martins RG, Stevenson JP, Jalal SI, et al: Carboplatin and pemetrexed with or without pembrolizumab for advanced, non-squamous non-small-cell lung cancer: A randomised, phase 2 cohort of the open-label KEYNOTE-021 study. Lancet Oncol 17: 1497-1508, 2016.

21. Schvartsman G, Peng SA, Bis G, Lee JJ, Benveniste MFK, Zhang J, Roarty EB, Lacerda L, Swisher S, Heymach JV, et al: Response rates to single-agent chemotherapy after exposure to immune checkpoint inhibitors in advanced non-small cell lung cancer. Lung Cancer 112: 90-95, 2017.

22. Szabados B, van Dijk N, Tang YZ, van der Heijden MS, Wimalasingham A, Gomez de Liano A, Chowdhury S, Hughes S, Rudman S, Linch M and Powles T: Response rate to chemotherapy after immune checkpoint inhibition in metastatic urothelial cancer. Eur Urol 73: 149-152, 2018.

23. Sonpavde G, Sternberg CN, Rosenberg JE, Hahn NM, Galsky MD and Vogelzang NJ: Second-line systemic therapy and emerging drugs for metastatic transitional-cell carcinoma of the urothelium. Lancet Oncol 11: 861-870, 2010.

24. Osa A, Uenami T, Naito Y, Hirata H, Koyama S, Takimoto T, Shiroyama T, Futami S, Nakatsubo S, Sawa N, et al: Monitoring antibody binding to $\mathrm{T}$ cells in a pembrolizumab-treated patient with lung adenocarcinoma on hemodialysis. Thorac Cancer 10: 2183-2187, 2019

25. National Comprehensive Cancer Network: Guidelines on bladder cancer. https://www.ncen.org/professionals/physician_gls/pdf/bladder.pdf. Accessed Jun 1, 2020.

26. Loriot Y, Necchi A, Park SH, Garcia-Donas J, Huddart R, Burgess E, Fleming M, Rezazadeh A, Mellado B, Varlamov S, et al: Erdafitinib in locally advanced or metastatic urothelial carcinoma. N Engl J Med 381: 338-348, 2019.

27. Rosenberg JE, O'Donnell PH, Balar AV, McGregor BA, Heath EI, Yu EY, Galsky MD, Hahn NM, Gartner EM, Pinelli JM, et al: Pivotal trial of enfortumab vedotin in urothelial carcinoma after platinum and anti-programmed death 1/programmed death ligand 1 therapy. J Clin Oncol 37: 2592-2600, 2019.

28. Roth BJ, Dreicer R, Einhorn LH, Neuberg D, Johnson DH, Smith JL, Hudes GR, Schultz SM and Loehrer PJ: Significant activity of paclitaxel in advanced transitional-cell carcinoma of the urothelium: A phase II trial of the Eastern cooperative oncology group. J Clin Oncol 12: 2264-2270, 1994.

29. Burch PA, Richardson RL, Cha SS, Sargent DJ, Pitot HC IV, Kaur JS and Camoriano JK: Phase II study of paclitaxel and cisplatin for advanced urothelial cancer. J Urol 164: 1538-1542, 2000.

30. Vaishampayan UN, Faulkner JR, Small EJ, Redman BG, Keiser WL, Petrylak DP and Crawford ED: Phase II trial of carboplatin and paclitaxel in cisplatin-pretreated advanced transitional cell carcinoma: A Southwest oncology group study. Cancer 104: 1627-1632, 2005.

31. Soga N, Onishi T, Arima K and Sugimura Y: Paclitaxel carboplatin chemotherapy as a second-line chemotherapy for advanced platinum resistant urothelial cancer in Japanese cases. Int J Urol 14: 828-832, 2007

32. Esteban-Fernández D, Verdaguer JM, Ramírez-Camacho R, Palacios MA and Gómez-Gómez MM: Accumulation, fractionation, and analysis of platinum in toxicologically affected tissues after cisplatin, oxaliplatin, and carboplatin administration. J Anal Toxicol 32: 140-146, 2008.

This work is licensed under a Creative Commons Attribution-NonCommercial-NoDerivatives 4.0 International (CC BY-NC-ND 4.0) License. 